\title{
Avaliação de um Treinamento de Habilidades Sociais (THS) com Universitários e Recém-Formados
}

\author{
Alessandra Turini Bolsoni-Silva \\ Universidade Estadual Paulista \\ Vanessa Barbosa Romera Leme \\ Universidade de São Paulo \\ Anne Midori Abe de Lima \\ Florêncio Mariano da Costa-Júnior \\ Marta Regina Gonçalves Correia \\ Universidade Estadual Paulista
}

\begin{abstract}
RESUMO
O objetivo desta pesquisa foi avaliar a efetividade de um treinamento de habilidades sociais, através de medidas de avaliação pré e pós-teste, com universitários e/ou recém-formados que apresentavam queixas interpessoais. Participaram 15 universitários e três recém-formados que procuraram o Centro de Psicologia Aplicada de uma universidade pública. O programa contou com vinte encontros semanais em grupo, distribuídos no ano letivo, com duração de duas horas cada; foram conduzidos seis grupos de intervenção com média de três participantes cada. Como medida de avaliação foi utilizado o IHS-Del-Prette, cujos dados foram tratados através de estatística não paramétrica (Teste Wilcoxon). Os resultados indicaram aquisições importantes apontando para a efetividade do procedimento de intervenção. De todo modo, os achados precisam ser relativizados devido ao número reduzido da amostra, ao uso do relato verbal como fonte primária de dados, pela ausência de grupo controle e de avaliação de seguimento, o que poderá ser testado em pesquisas futuras.
\end{abstract}

Palavras-chave: treinamento de habilidades sociais; estudantes universitários; intervenção psicológica; prevenção.

\begin{abstract}
Evaluation of Social Skills Training with College Students and Recently Graduates

The objective of this research was to evaluate the effectiveness of a social skills training, through pre and post-test evaluation measurements, in a group of college students and/or recent graduates who showed interpersonal complaints. Fifteen college students and three recent graduates, who came to the Center for Applied Psychology in a public university, participated in the training. The program consisted of a series of 20 weekly group-encounters over the academic year, and each session was two hours long; six intervention groups were conducted with an average of three participants in each group. The IHS-Del-Prette was used as measurement instrument, and the data were treated through a non-parametric statistical test (Wilcoxon Test). The results indicated important acquisitions which led to the effectiveness of the intervention procedure. However, the results must be analyzed considering the small sample size, the use of oral reports as the main data source and also the absence of a control or follow-up group, which can be tested in future studies.
\end{abstract}

Keywords: social skills training; college students; psychological intervention; prevention.

A literatura aponta para relação inversa entre repertório de habilidades sociais e problemas psicológicos para o estudante universitário. Por exemplo Furtado, Falcone e Clark (2003) encontraram que quanto menos frequentes são as habilidades sociais, maior o estresse. É notória a influência de cursos de graduação sobre a assertividade e as habilidades sociais (Del Prette \& Del Prette, 1983).

Atualmente, não se dispõe de um conceitual que defina habilidades sociais de uma maneira unívoca e consensual. Uma possibilidade é definir habilidades sociais como um conjunto de comportamentos emiti- 
dos pelo indivíduo diante das demandas de uma situação interpessoal (Del Prette \& Del Prette, 1999). Del Prette e Del Prette (2001a) propuseram as seguintes definições para formas de interagir socialmente:

... [reação] habilidosa (que demonstra assertividade, empatia, expressão de sentimentos positivos ou negativos de forma apropriada, civilidade etc.), [reação] não habilidosa passiva (que demonstra esquiva ou fuga ao invés de enfrentamento da situação) e [reação] não habilidosa ativa (que demonstra agressividade, negativismo, ironia, autoritarismo etc.) (p. 65).

A aquisição das habilidades sociais, dentro do contexto universitário, ocorre às vezes de forma "oculta", ou seja, ao surgirem as novas demandas, os indivíduos tentam se adaptar sozinhos, porém, há pessoas que apresentam dificuldades acentuadas tornando-se desamparadas por não conseguirem aumentar seus repertórios sociais (Pacheco \& Rangé, 2006). Furtado e cols. (2003) e Cole, Lazarick e Howard (1986) afirmam que as vivências acadêmicas, quando não garantem uma boa qualidade de vida, tornam-se experiências estressantes e até depressivas, além de influenciar no rendimento acadêmico. Essas dificuldades também podem resultar na diminuição da autoestima, aumento da ansiedade, fracasso nas atividades acadêmicas ou dificuldade para concluir o curso iniciado; além desses fatores, tais déficits interpessoais, também podem contribuir para o engajamento em comportamentos de risco como o uso de drogas (Bandeira \& Quaglia, 2005; Botvin \& Wills, 2008; Glasgow \& Caul, 2008; Inglés, Hidalgo \& Mendéz, 2005; Magalhães \& Murta, 2003).

Enquanto repertório requerido para bom desempenho na universidade e relatado como difícil de ser realizado, podem ser destacadas as habilidades sociais envolvidas no falar em público (apresentação de seminários, responder perguntas de professor, fazer comentários ou dar recados em sala de aula, falar com autoridade, reclamar com o professor sobre notas e avaliações, trabalhar em grupo: ouvir, concordar/discordar, lidar com críticas, negociar, argumentar, perguntar, responder perguntas), para lidar com relacionamentos amorosos - abordar para relação amorosa, manter ou terminar relacionamentos (Boas, Silveira \& Bolsoni-Silva, 2005; Del Prette \& Del Prette, 2003; Del Prette \& cols., 2004; Del Prette, Del Prette \& Barreto, 1998; Pacheco \& Rangé, 2006) - e na interação com familiares (Bandeira \& Quaglia, 2005). Adicionalmente, pode-se destacar pesquisas que comparam e/ou descrevem o repertório de habilidades sociais, independente do contexto. Del Prette e cols. (2004), com uma amostra de 564 estudantes de psicologia, identificaram maior frequência de habilidades assertivas (Fator 1), de conversação e desenvoltura social (Fator 3) e de auto-exposição a desconhecidos e situações novas (Fator 4) e menor frequência em expressão de afeto positivo e de auto-controle da agressividade (Fator 5). Bandeira e Quaglia (2005), ao estudarem 40 universitários, encontraram queixas relacionadas a situações negativas de expressar insatisfação e de solicitar mudança de comportamento, especialmente em situações de interações com familiares.

Deve-se ressaltar que a aquisição das habilidades sociais também é importante para a vida profissional do indivíduo após o término da faculdade, por isso torna-se imprescindível que o treinamento garanta generalização dos comportamentos socialmente habilidosos para diversas situações sociais (Del Prette \& cols., 1998). Reiterando, na opinião de Del Prette e Del Prette (1983), os atuais processos de trabalho são alicerçados na natureza e na qualidade das relações interpessoais e exigem, além das competências técnicas, a competência social nas interações profissionais.

Quanto ao uso do Treinamento em Habilidades Sociais (THS) com universitários, na literatura internacional, pode-se afirmar que são dirigidos ao uso de substâncias e à fobia social. As pesquisas utilizam delineamento de grupo experimental (Cozby, 2003), com amostras acima de 100 participantes e atestam para a efetividade do THS quanto à redução de ansiedade e fobia social (Herbert, Rheingold \& Goldstein, 2002; Mersch, 1992; Wlazlo, Schroeder-Hartwig, Hand, Kaiser \& Münchau, 2005). Por outro lado, Gorman (1996), ao fazer um estudo de revisão sobre a efetividade do THS na redução do uso do álcool, afirma que a maioria das pesquisas indica insucesso do procedimento. A partir dos estudos apresentados pode-se concluir que o déficit de habilidades sociais pode gerar ansiedade, fobia social, dificuldades acadêmicas e uso de drogas, mas não o uso de álcool por universitários, sugerindo que para essa problemática faz-se necessário outro procedimento de intervenção.

Para descrever a produção em habilidades sociais no Brasil, Bolsoni-Silva e cols. (2006) analisaram 55 artigos empíricos, até o ano de 2004, encontrando estudos que buscaram caracterizar populações, correlacionar variáveis e desenvolver instrumentos. No caso dos universitários, 11 estudos $(20 \%)$ foram conduzidos e apenas dois deles testaram a efetividade de intervenções (Del Prette \& Del Prette, 2003; Falcone, 1999). Após esse período, no Brasil, na forma de arti- 
go, encontram-se o relato dos estudos de Magalhães e Murta (2003), o qual se encontrava no prelo na época da publicação mencionada e o de Boas e cols. (2005).

Magalhães e Murta (2003) intervieram em um grupo de 13 estudantes de psicologia, após divulgação na universidade, que foram distribuídos em quatro subgrupos, valendo-se do IHS-Del-Prette como medida de pré e pós-teste. As habilidades sociais foram treinadas em 10 sessões, a partir de discussões teóricas e técnicas cognitivas-comportamentais e envolviam lidar com emoções, defender os próprios direitos, comunicar-se, falar em público, elogiar, lidar com críticas e manejo de raiva. Ainda que não tenha utilizado tratamento estatístico, os resultados sinalizaram melhora em todos os Fatores avaliados no IHS-DelPrette, com exceção do Fator 5 que corresponde a auto-controle da agressividade. Del Prette e Del Prette (2003) também utilizaram o delineamento A-B-A, com duas sessões semanais de duas horas cada, em um total de 17 encontros, a partir de discussões teóricas e técnicas cognitivas-comportamentais. O objetivo do estudo foi o treino de habilidades sociais no trabalho com 10 estudantes da área de exatas: busca de emprego e atuação profissional. Para tanto teve por foco o treino em observar, expressar sentimentos e empatia, prover feedback, elogiar, comunicar-se, falar em público e expor próprias opiniões. Os resultados indicaram melhora significativa em todos os fatores avaliados no IHS-Del-Prette. Em outra pesquisa, Falcone (1999) descreveu um programa de treinamento de empatia com um grupo de 10 universitários, após divulgação na universidade. A intervenção consistiu de 11 encontros de duas horas cada e também teve um grupo controle de sete participantes. Utilizou filmagem como medida de avaliação e se propôs a ensinar a definição de empatia e de como se comportar empaticamente, sobretudo por técnicas cognitivas. Os resultados mostraram melhora tanto na comunicação verbal como na não-verbal relacionada à empatia. Já o trabalho de Boas e cols. (2005) foi um estudo piloto, com quatro participantes, do procedimento avaliado neste artigo, prevendo o treino de comportamentos socialmente habilidosos usando apenas técnicas comportamentais. A intervenção compreendeu 22 sessões que ocorreram uma vez por semana, com duas horas de duração, distribuídas no período letivo; houve avaliação pré e pós-teste através de entrevista clínica (Bolsoni-Silva, Bitondi \& Marturano, 2008) e do IHSDel-Prette. Os universitários relataram superação das seguintes dificuldades: fazer elogios, iniciar e manter conversação, expor-se a situações sociais, discordar, autoconhecer-se e redução de sintomas de ansiedade que prejudicavam o dormir e favoreceriam o uso de bebida alcoólica. Por outro lado, todos os quatro apresentavam indicação clínica para atendimento, conforme o IHS-Del-Prette, o que não foi superado após a intervenção, sugerindo a necessidade de aprimoramento do procedimento a fim de obter melhores resultados com a população estudada.

Ao analisar os estudos de intervenção nacionais (Boas \& cols., 2005; Del Prette \& Del Prette, 2003; Falcone, 1999; Murta \& Magalhães, 2003) conclui-se que: a) utilizaram o IHS-Del-Prette como medida de avaliação pré e pós-intervenção, com exceção de Falcone (1999); b) utilizaram técnicas cognitivo-comportamentais, com exceção de Boas e cols. (2005) que se valeu de técnicas comportamentais; c) três utilizaram delineamento de grupo quase-experimental (Boas \& cols., 2005; Del Prette \& Del Prette, 2003; Murta \& Magalhães, 2003) e um experimental (Falcone,1999); e) relataram sucesso através de sessões de grupo, ainda que com limitações devido ao baixo número de participantes e o controle de variáveis; f) o número de sessões variou de 10 (Falcone, 1999; Magalhães \& Murta, 2003) para próximo a 20 (Boas \& cols., 2005; Del Prette \& Del Prette, 2003); g) nenhuma dessas pesquisas se propôs a treinar habilidades sociais nos três contextos de dificuldade para o universitário: interação com colegas/professores, relacionamento amoroso e familiar.

Os estudos descritos utilizam instrumentos de autorrelato como preditores de comportamento, os quais, segundo Gorecki, Dickson, Anderson e Jones (1981) possuem limitações, uma vez que o relato pode ser discrepante do que as pessoas fazem em situação natural, ainda que na situação de role-playing, em setting terapêutico, foi encontrada correspondência entre $o$ fazer e dizer. O relato pode ter ainda mais problema quando as pessoas possuem ansiedade social, que tendem a avaliar mais negativamente seu desempenho do que de fato o fizeram (Norton \& Hope, 2001). Por outro lado há autores que defendem o uso de instrumentos de relato, por exemplo Glass e Kellner (1987) afirmam que a utilização de escalas de autorrelato com informações quantitativas trouxe um grande avanço científico, por serem livres de preconceitos do experimentador e também por serem econômicas quanto a tempo e esforço. Snaith (1981) reitera a relevância de questionários e de escalas para mensurar a gravidade dos problemas e acrescenta que servem também para detectar a incidência de transtorno na comunidade e estabelecer padrões de sintomas ou de 
outras características do paciente e de seus familiares. Concluindo, o uso de autorrelato como medida de avaliação pode ser utilizada, mas os resultados obtidos precisam ser relativizados, sendo o ideal a combinação de relato e de observação.

Considerando os estudos de caracterização (Bandeira \& Quaglia, 2005; Del Prette \& cols., 2004; Del Prette \& Del Prette, 2003; Pacheco \& Rangé, 2006) e os de intervenção (Boas \& cols., 2005; Del Prette \& Del Prette, 2003; Falcone, 1999; Magalhães $\&$ Murta, 2003) julga-se importante testar um procedimento: a) com maior número de participantes; b) que busque promover as habilidades sociais indicadas como importantes pela literatura: comunicação, expressar sentimentos positivos e elogiar, direitos humanos, conhecer sobre diferentes formas de se comportar (habilidoso e não habilidoso), expressar sentimento negativo e discordar, lidar com críticas, os quais envolvem o controle da agressividade nos três contextos relevantes para o universitário (interação com colegas/professores, relacionamento amoroso e familiar).

Diante dessas considerações, o presente estudo avalia, através de medidas pré e pós-teste, um programa de intervenção em grupo cuja finalidade foi a de promover habilidades sociais em estudantes universitários e recém-formados com dificuldades interpessoais.

\section{MÉTODO}

\section{Participantes}

Participaram deste estudo 15 estudantes universitários e três recém-formados de uma cidade no interior do estado de São Paulo, com queixas e dificuldades interpessoais na área das habilidades sociais. A amostra foi composta por 10 participantes do sexo masculino e oito do sexo feminino com idades entre 19 e 37 anos (média: 22,3; dp: 5,4). Dentre os participantes da amostra, cinco cursavam Engenharia, quatro Jornalismo, um Educação Física, três Relações Públicas, um Serviço Social, um Desenho Industrial e um História.

\section{Instrumentos}

1. Roteiro de entrevista semi-estruturada. O objetivo da realização dessa entrevista é o de receber os estudantes, informar sobre o atendimento em grupo e coletar dados sobre as queixas e variáveis relacionadas. Essa entrevista foi utilizada previamente com pais que buscaram atendimento, mostrando-se efetiva na obtenção de dados dessa natureza (Bolsoni-Silva, de Paiva \& Barbosa, 2009). A entrevista é composta das seguintes questões norteadoras:

$\checkmark$ Como soube do grupo?

$\checkmark \mathrm{O}$ que te fez pensar em procurar o grupo? Se esta pergunta levar à descrição da queixa:

$\checkmark$ Quantas vezes esse comportamento acontece? Desde quando acontece? Em que situações? Onde você estava? O que estava fazendo anteriormente? Quando isso ocorre o que você faz? $\mathrm{O}$ que acontece depois?

Se esta pergunta não levar à descrição da queixa:

$\checkmark$ Fale um pouco sobre seu relacionamento com seus amigos na faculdade? Você encontra dificuldades para se relacionar com eles? Porque você acha que estas dificuldades ocorrem? Você poderia me dar alguns exemplos?

O terapeuta deve fazer outras perguntas de forma a operacionalizar a queixa e colher dados acerca de variáveis relacionadas.

2. Inventário de Habilidades Sociais - IHS-DelPrette (Del Prette \& Del Prette, 2001b), o qual caracteriza o desempenho social em situações cotidianas. $\mathrm{O}$ instrumento é composto por 38 itens que apresentam ações e sentimentos frente a determinadas situações, diante das quais o participante deve avaliar a frequência com que age ou sente: nunca ou raramente (escore 0 ); com pouca frequência (escore 1); com regular frequência (escore 2); muito frequentemente (escore 3 ); sempre ou quase sempre (escore 4). Entre os fatores estão: Fator 1: enfrentamento e auto-afirmação com risco; Fator 2: auto-afirmação na expressão de sentimento positivo; Fator 3: conversação e desenvoltura social; Fator 4: auto-exposição a desconhecidos e situações novas; Fator 5: autocontrole da agressividade; adicionalmente o IHS-Del-Prette conta com um conjunto de itens denominados de "itens que não entraram em nenhum fator" (Outros), os quais são: negociar uso de preservativo, pedir mudança de conduta, lidar com críticas justas, expressar desagrado a amigos, recusar pedido abusivo, interromper a fala do outro. O IHS-Del-Prette possui dados normativos para os escores fatoriais e o escore total, em percentis, para a amostra feminina e a masculina, que permitem interpretar os resultados em: a) repertório bastante elaborado de habilidades sociais $(80 \%$ a $100 \%)$; bom 
repertório de habilidades sociais - acima da média (55\% a $75 \%)$; repertório médio $(50 \%)$; bom repertório de habilidades sociais - abaixo da média $(30 \%$ a $45 \%$ ); indicação para treinamento em habilidades sociais $(1 \%$ a $25 \%)$. O instrumento possui validação para a população universitária (Bandeira, Costa, Del Prette, Del Prette \& Gerk-Carneiro, 2000), apresentando valores de referência para a classificação diagnóstica.

\section{Procedimentos de coleta e análise de dados}

A amostra foi recrutada através de divulgação do programa nas universidades (pública e privadas da cidade) e de cartazes afixados nos campi e de visitas às salas de aula (com exceção dos cursos de psicologia). A rádio universitária também divulgou o programa de intervenção para a comunidade em geral e a coordenadora do projeto foi entrevistada. No momento da divulgação, especificou-se que o grupo destinava-se a estudantes com dificuldades quanto ao relacionamento interpessoal e à adaptação ao contexto da universidade. As pessoas que se inscreveram foram entrevistadas acerca das queixas que motivaram a busca pelo atendimento e então lhes foi explicado sobre a intervenção e sobre a pesquisa resultante da mesma, momento em que foi solicitada a assinatura do Termo de Consentimento Livre e Esclarecido (TCLE), que constava informações sobre o objetivo do trabalho, garantindo o respeito aos procedimentos previstos na Resolução 196/96. As pessoas, que ao serem avaliadas pelo grupo de pesquisa apresentavam dificuldades interpessoais em pelo menos um contexto (vida acadêmica, relacionamento amoroso e/ou relacionamento familiar) foram encaminhadas aos grupos de intervenção. Foram conduzidos seis sub-grupos de intervenção, no período de 2005 a 2007, com média de 3 participantes cada. Os participantes foram avaliados, através do IHS-Del-Prette, antes e após a intervenção. Para tanto, utilizou-se delineamento de grupo, pois o procedimento aplicado aos seis sub-grupos foi estruturado previamente (similar a Bolsoni-Silva, 2009a) e supervisionado pela mesma professora, ainda que os estagiários (terapeutas e co-terapeutas) tenham sido diferentes em cada subgrupo. Os dados foram comparados com auxílio de um pacote estatístico (SPSS versão 8.0) através do Teste Wilcoxon. Os participantes também foram classificados em clínicos e não clínicos conforme classificação no IHS-Del-Prette, nas duas medidas de avaliação, cujas informações encontram-se no corpo do texto.

\section{O Programa de Intervenção}

Inicialmente, os participantes passaram por uma entrevista semi-estruturada aplicada individualmente (Bolsoni-Silva \& cols., 2008) e, na sequência houve a aplicação do IHS-Del-Prette. A análise de ambos os instrumentos permitiu elaborar um estudo de caso individualizado que norteou objetivos para cada participante e para o grupo durante todo o procedimento. A análise da entrevista, além de auxiliar na elaboração do estudo de caso, permitiu checar acerca dos critérios de inclusão no estudo, isto é, se o participante apresentava dificuldades em pelo menos um dos três contextos: vida acadêmica, relacionamento amoroso e/ou relacionamento familiar. A análise da entrevista constatou que as queixas dos participantes estavam relacionadas diretamente ao contexto universitário $(\mathrm{n}=18)$, seja quanto a falar em público $(\mathrm{n}=13)$, seja para fazer amizades $(\mathrm{n}=9)$; um número menor de participantes também se queixou do relacionamento familiar $(n=5)$ e do relacionamento amoroso $(n=6)$.

As intervenções foram realizadas na clínica-escola da universidade e consistiram em 20 encontros, uma vez por semana, com aproximadamente duas horas de duração cada, cujos temas encontram-se na Tabela 1 . As sessões foram conduzidas por dois terapeutas, que alternavam na função de terapeuta e co-terapeuta, as quais foram gravadas para posterior transcrição.

As sessões tiveram uma estrutura programada em todos os encontros. Cada tarefa era discutida no início da sessão, a partir de medidas de auto-registro e, em seguida, explicitado o tema do encontro. Nesse momento era realizada uma exposição dialogada do tema relacionando com o que o grupo mencionava quanto às suas dificuldades. Tanto no momento da tarefa quanto na exposição do tema, as situações relatadas pelos participantes eram investigadas pelos terapeutas, os quais buscavam identificar variáveis envolvidas nas queixas e elaboravam, junto aos participantes, alternativas de resolução para as suas dificuldades. O passo subsequente consistia no treino da habilidade alvo da sessão, através de role playing e/ou atividades de discussão, ocasião em que respostas eram modeladas a partir da colaboração do grupo, utilizando diversas técnicas comportamentais, tais como modelagem, instrução e reforçamento diferencial. Ao final de cada sessão, os participantes faziam uma avaliação do encontro, realizada ora de maneira escrita, ora verbalmente e recebiam uma tarefa de casa. Os participantes recebiam uma cartilha que continha (similar a Bolsoni-Silva, 2009b) para cada encontro, definições 
das habilidades sociais e exemplos de sua aplicabilidade para os contextos de vida universitária, relacionamento amoroso e familiar. Pela Tabela 1 nota-se que até a sessão 11 e na sessão 14 os temas foram trabalhados para os três contextos simultaneamente e também houve sessões específicas para a vida universitária (sessões 13,16,17, 19 e 20), relacionamento amoroso (sessão 12) e relacionamento familiar (sessão $18)$.

TABELA 1

Descrição das Sessões e dos Temas Trabalhados

\begin{tabular}{ll}
\hline SESSÕES & TEMAS \\
\hline Sessão 1 & Comunicação: iniciar e manter conversações \\
Sessão 2 & Comunicação: fazer e responder perguntas \\
Sessão 3 e 4 & Expressar sentimento positivo, elogiar, dar/receber feedback positivo, agradecer \\
Sessão 5 & Conhecer direitos humanos básicos \\
Sessão 6 & Expressar e ouvir opiniões (de concordância, de discordância) \\
Sessão 7 & Conhecer comportamento habilidoso e não habilidoso (ativo e passivo) \\
Sessão 8 & Expressar sentimentos negativos, dar e receber feedback negativo \\
Sessão 9 & Fazer e recusar pedidos \\
Sessão 10 e 11 & Lidar com críticas, admitir próprios erros, pedir desculpas \\
Sessão 12 & Não-verbal, relacionamento amoroso \\
Sessão 13 & Falar em público \\
Sessão 14 & Resolver problemas \\
Sessão 15 & Expressar empatia \\
Sessão 16 & Interagir com autoridade \\
Sessão 17 & Liderança \\
Sessão 18 & Dialogar com pais, relacionamentos familiares \\
Sessão 19 & Trabalhar em equipe \\
Sessão 20 & Fazer amizades \\
\hline
\end{tabular}

\section{RESULTADOS}

São apresentados, nesta seção, os resultados das medidas pré e pós-teste do IHS-Del-Prette que apresentaram diferença significativa. Em seguida, são descritos os resultados referentes à classificação diagnóstica do IHS-Del-Prette.

A Tabela 2 apresenta os resultados das comparações das medidas pré e pós-teste dos itens e dos fatores do IHS-Del-Prette com diferença significativa. Demonstra que houve aumento nas médias do pós-teste em relação às do pré-teste, com diferença significativa, em 12 dos $38(31,6 \%)$ itens avaliados no IHS-Del-Prette, em 3 dos 5 Fatores (60\%) e no Escore total $(\mathrm{p}=0,001)$. Os dados indicam, através do aumento das médias no pós-teste que todos os partici- pantes obtiveram ganhos com a intervenção quanto às habilidades sociais (manter conversa com desconhecidos, cobrar dívida de amigo, apresentar-se a outra pessoa, abordar para relação sexual e discordar do grupo) referentes ao Fator 1 (Enfrentamento e autoafirmação com risco). Em seguida, o Fator 3 (Conversação e desenvoltura social) indica ganhos nas habilidades sociais abordar autoridade e manter conversação. Já o Fator 4 (Auto-exposição a desconhecidos e situações novas) apresenta ganhos nas habilidades sociais falar a público desconhecido, fazer pergunta a desconhecido e pedir favores a desconhecido. Por fim, os resultados sinalizam ganhos quanto à habilidade social expressar desagrado e de pedir ajuda a amigos referente ao item Outros. 
TABELA 2

Médias das Medianas Pré e Pós-teste dos Itens e dos Fatores do IHS-Del-Prette com Diferença Significativa (Teste Wilcoxon)

\begin{tabular}{|c|c|c|c|}
\hline \multirow{2}{*}{ Itens } & \multicolumn{2}{|c|}{ Médias } & \multirow[b]{2}{*}{$P^{*}$} \\
\hline & pré-teste & pós-teste & \\
\hline \multicolumn{4}{|l|}{ F1- Enfrentamento e auto-afirmação com risco } \\
\hline 01 - Manter conversa com desconhecidos & 0,94 & 2,17 & 0,002 \\
\hline 05 - Cobrar dívida de amigo & 1,89 & 2,94 & 0,007 \\
\hline 07 - Apresentar-se a outra pessoa & 1,11 & 1,61 & 0,050 \\
\hline 12 - Abordar para relação sexual & 1,11 & 1,83 & 0,013 \\
\hline 16 - Discordar do grupo & 1,94 & 2,61 & 0,005 \\
\hline Total F1 & 21,39 & 28,33 & 0,001 \\
\hline \multicolumn{4}{|l|}{ F3 - Conversação e desenvoltura social } \\
\hline 19 - Abordar autoridade & 1,22 & 2,50 & 0,006 \\
\hline 36 - Manter conversação & 2,67 & 3.11 & 0,019 \\
\hline Total F3 & 15,11 & 19,06 & 0,031 \\
\hline \multicolumn{4}{|c|}{ Fator 04 - Auto-exposição a desconhecido e situações novas } \\
\hline 09 - Falar a público desconhecido & 2,22 & 3,22 & 0,011 \\
\hline 23 - Fazer pergunta a desconhecido & 2,06 & 3,33 & 0,003 \\
\hline 26 - Pedir favores a desconhecidos & 2,22 & 2,94 & 0,050 \\
\hline Total F4 & 9,44 & 12,72 & 0,003 \\
\hline \multicolumn{4}{|l|}{ Outros } \\
\hline 27 - Expressar desagrado a amigos & 2,56 & 3,50 & 0,027 \\
\hline 32 - Pedir ajuda a amigos & 1,83 & 2,72 & 0,018 \\
\hline Total Outros & 16,33 & 20,06 & 0,001 \\
\hline Escore total & 86,56 & 106,89 & 0,001 \\
\hline
\end{tabular}

${ }^{*} p \geq 0,05$

Os itens sem diferença significativa são: a) Fator 1 (devolver mercadoria defeituosa, lidar com críticas injustas, fazer perguntas a desconhecidos, declarar sentimento amoroso, discordar de autoridade e falar a público conhecido); b) Fator 2 (agradecer elogios, elogiar familiares, participar de conversação, expressar sentimento positivo, defender outrem em grupo, elogiar outrem, expressar sentimento positivo); c) Fator 3 (encerrar conversação, pedir favores a colegas, reagir a elogio, recusar pedidos abusivos e encerrar conversa ao telefone); d) Fator 4 (falar a público conhecido); e) Fator 5 (lidar com críticas dos pais, lidar com chacotas e cumprimentar desconhecidos); f) Outros (negociar uso de preservativo, pedir mudança de conduta, lidar com críticas justas, recusar pedido abusivo e interromper a fala do outro).

A análise da classificação dos universitários no IHS-Del-Prette indicou que metade dos participantes $(\mathrm{n}=9)$ apresentou, antes da intervenção, necessidade de treinamento em habilidades sociais; já os demais participantes apresentavam um bom repertório de habilidades sociais, o que foi mantido no pós-teste. Após a intervenção, dos 9 com indicativo clínico, apenas um participante permaneceu com tal indicação, um piorou sua classificação e os demais $(n=7)$ melhoraram sua classificação no pós-teste do IHS-DelPrette.

Ao relacionar os itens que apresentaram diferença significativa (Tabela 2) com os contextos de interação, podem-se identificar melhoras nas seguintes habilidades: a) Relacionamento com colegas/professores: cobrar dívida de amigo, discordar do grupo, abordar autoridade, pedir ajuda a amigos, falar a público desconhecido e expressar desagrado a amigos; b) Relacionamento amoroso: apresentar-se a outra pessoa e 
abordar para relação sexual. As habilidades que envolviam relacionamento com familiares (por exemplo, elogiar familiares e lidar com críticas dos pais) não indicaram resultados significativos no IHS-Del-Prette, após a intervenção.

\section{DISCUSSÃO}

Os resultados encontrados nas avaliações pré e pós-teste sugerem que o THS foi eficaz ao desenvolver um conjunto de comportamentos referentes às queixas relatadas pelos participantes, ainda que algumas não puderam ser superadas. $\mathrm{O}$ programa pode ser considerado como preventivo, na medida em que foi divulgado e composto por participantes que não necessariamente estavam na lista de espera da clínica-escola da universidade e que também não apresentavam diagnóstico para transtornos de ansiedade e depressão. Contudo, metade dos participantes teve indicação clínica para o THS nas pré-avaliações, sinalizando a importância para os psicólogos e pesquisadores em criar demanda de intervenção. Nesse caso, os dados sinalizaram que houve superação de parte das dificuldades para a grande maioria dos participantes. Resultados semelhantes são observados em outros programas de Treinamento de Habilidades sociais com universitários (Magalhães \& Murta, 2003; Pacheco \& Rangé, 2006), os quais também se caracterizavam como preventivos e tiveram melhoras no repertório de seus participantes. É importante destacar que apenas um dentre os 18 participantes permaneceu, no pós-teste, com indicação clínica de atendimento no que tange ao escore total do IHS-Del-Prette, o que indica que o procedimento de intervenção em grupo pode ser econômico como uma primeira forma de atendimento. Entretanto, seria interessante que fossem oferecidos atendimentos individuais para aqueles que não conseguirem superar suas principais dificuldades. Outra possibilidade seria prolongar o treinamento de habilidades sociais ou ainda oferecer simultaneamente o atendimento em grupo e individual para aqueles com maiores dificuldades dianósicas no pré-teste.

Ao analisar especificamente os resultados de cada fator do IHS-Del-Prette, nota-se que o THS promoveu ganhos estatisticamente significativos para a maioria das habilidades que compõem o Fator 1, o qual corresponde à habilidade de enfrentamento e auto-afirmação com risco, isto é, lidar com situações interpessoais, que necessitem da afirmação de seus próprios direitos, preservando sua auto-estima em situações que apresentem a possibilidade de reação indesejável do interlocutor. Outros fatores que apresentaram o desenvolvimento significativo do repertório de habilidades sociais foram: a) o Fator 4 (auto-exposição a desconhecidos e situações novas); b) Fator 3 (conversação e desenvoltura social); c) Outros (expressar desagrado e pedir ajuda a amigos). Estes achados vão ao encontro de outros estudos (Boas \& cols., 2005; Magalhães \& Murta, 2003; Pacheco \& Rangé, 2006), os quais indicaram que o THS pode obter resultados positivos em habilidades que não requeiram lidar com situações que envolvam expressão de sentimentos positivos, negativos e com risco potencial de reações indesejáveis, como o pedido de mudança de comportamento. Por outro lado, a literatura (Bandeira \& Quaglia, 2005; Del Prette \& cols., 2004) demonstra que essas dificuldades interpessoais são as mais relevantes para os universitários; e como os procedimentos preventivos (Boas \& cols., 2005; Magalhães \& Murta, 2003) parecem não ter superado inteiramente tais dificuldades, bem como a pesquisa relatada neste artigo, novos procedimentos precisam ser elaborados e testados. O estudo de Del Prette e Del Prette (2003) mostrou-se efetivo em todos os fatores avaliados, mas foi direcionado a população específica (universitários de exatas) e a tema específico (aplicabilidade para o trabalho), o que talvez tenha influenciado na obtenção de melhores resultados. Na literatura internacional e nacional, os estudos com THS também são direcionados ou a estudantes com fobia social (Herbert \& cols., 2002; Mersch, 1992; Wlazlo \& cols., 2005) ou com abuso de substâncias (Bandeira \& Quaglia, 2005; Botvin \& Wills, 2008; Glasgow \& Caul, 2008; Inglés \& cols., 2005; Magalhães \& Murta, 2003). Os estudos acerca da fobia social mostraram-se efetivos, o que não ocorreu quanto ao uso de substâncias (Gorman, 1996). O uso de técnicas ou o número de sessões são variáveis que não parecem determinantes na obtenção de resultados, pois as intervenções cognitivo-comportamentais com 10 sessões (Magalhães \& Murta, 2003) e as comportamentais com 20 sessões (Boas \& cols., 2005), obtiveram resultados semelhantes e em consonância com os achados obtidos na presente pesquisa. Pode-se afirmar ainda que os estudos internacionais possuem objetivos distintos dos nacionais. Os internacionais voltam-se para a redução de problemas psicológicos como fobia e uso de substâncias. Os nacionais visam o treino de habilidades sociais para melhorar relacionamentos no trabalho (Del Prette \& Del Prette, 2003), promover empatia (Falcone, 1999) ou interações sociais de maneira geral (Boas \& cols., 2005, Magalhães \& Murta, 2003), ainda que não tenham focado, enquanto 
temas dos encontros, os três contextos de dificuldade para o universitário (colegas/professores, relacionamento amoroso e familiar).

Nesse aspecto, o presente estudo destaca-se dos demais ao indicar que a intervenção foi benéfica ao proporcionar aos participantes ganhos nos repertórios de habilidades sociais referentes ao contexto de relacionamento universitário (colegas e professores) e amoroso, os quais foram identificados como queixas iniciais. Todavia, os dados sugeriram que os participantes não ampliaram as habilidades sociais referentes ao relacionamento com familiares. Essas habilidades compõem especificamente os Fatores 2 e 5; ambos não apresentaram diferenças significativas nas avaliações pré e pós-teste. Uma explicação para estes achados é o fato dos estudantes já não estarem vivendo com seus familiares, por ocasião da intervenção, o que pode ter diminuído as possibilidades de interação com tais pessoas e consequente ampliação de comportamentos; outra hipótese adicional é a de o tempo da intervenção seja insuficiente para captar ou instituir tais mudanças; uma terceira hipótese é a de seja insuficiente apenas uma sessão que tenha por tema o relacionamento com familiares no que tange a desenvolver comportamentos de resolução de problemas nesse contexto.

Desse modo, é pertinente que futuras intervenções invistam mais tempo com essas queixas que, de forma geral, envolvem um conjunto complexo de comportamentos, tais como, ter auto-controle, expressar sentimentos positivos e negativos. Nessa direção, Bandeira e Quaglia (2005) afirmam que expressar insatisfação e solicitar mudança de comportamento são as situações de maior desconforto, sobretudo nas interações familiares, o que está em consonância com os resultados da presente pesquisa. Uma outra interpretação viável é a de que, apesar do procedimento ter se pautado em avaliações das queixas individuais, é possível que o formato grupal com procedimento estruturado não tenha permitido a exaustão das demandas e déficits de cada participante. Estudos futuros que combinem atendimento individual com grupal poderiam responder melhor a esta questão. Também vale ressaltar a necessidade de avaliação de seguimento, para verificar se as habilidades de auto-controle da agressividade e expressão de sentimentos positivos foram generalizadas e mantidas para o contexto da vida real. Nesse sentido é relevante utilizar também outras medidas de avaliação, além de autorrelato, pois se sabe das dificuldades em seu uso, uma vez que nem sempre o relato corresponde ao que as pessoas fazem nas intera- ções sociais (Gorecki \& cols., 1981; Norton \& Hope, 2001).

Acrescenta-se que apesar do THS, do ponto de vista estatístico, não tenha promovido habilidades de expressão de sentimentos positivos e de auto-controle da agressividade, outras relevantes para o contexto de relacionamento amoroso e universitário foram desenvolvidas. Dentre essas, podemos citar as habilidades necessárias para relacionar-se com o sexo oposto, como se apresentar a outra pessoa e abordar para relação sexual; e as habilidades necessárias para a convivência com amigos e colegas, realizações de trabalhos em grupo e apresentações em seminários, tais como abordar autoridades, iniciar e manter conversação, cobrar dívidas, falar a público desconhecido, fazer perguntas e pedir favores a desconhecidos, o que tem respaldo na literatura (Boas \& cols., 2005; Del Prette \& cols., 1998; Del Prette \& cols., 2004; Del Prette \& Del Prette, 2003; Pacheco \& Rangé, 2006) e que pode prevenir problemas psicológicos e abuso de substâncias (Bandeira \& Quaglia, 2005; Botvin \& Wills, 2008; Cole \& cols., 1986; Furtado \& cols., 2003; Glasgow \& Caul, 2008; Inglés \& cols., 2005; Magalhães \& Murta, 2003).

Os resultados encontrados são importantes, mas precisam ser relativizados diante da metodologia empregada que envolve o uso de relato, além de ser um estudo quase-experimental. Quanto ao uso de autor-relato há de considerar também a variável desejabilidade social, ou seja, as pessoas ao participarem da intervenção podem ter aprendido sobre o que se esperava que respondessem na avaliação de pós-teste, cujas respostas podem não condizer ao desempenho em contexto natural. Por outro, como o IHS-Del Prette possui 15 de 38 itens com fraseado negativo, há uma minimização do efeito dessa desejabilidade, dificultando o falseamento das respostas (Welter \& Capitão, 2007).

\section{CONSIDERAÇÕES FINAIS}

Os resultados do presente estudo indicam que o procedimento pode ser útil para desenvolver habilidades sociais e avança no conhecimento da área uma vez que envolve um número maior de participantes, com distribuição equitativa entre homens e mulheres. Um aspecto a ser considerado diz respeito ao potencial de prevenção do procedimento uma vez que foi procurado por estudantes sem indicação clínica, os quais apresentavam déficits em habilidades sociais e que melhoraram estatisticamente após a intervenção. 
Estudos futuros podem combinar outras medidas de avaliação, como a observação, grupo controle e seguimento. Os resultados, que estão em consonância com a literatura, indicam que o THS sempre traz resultados positivos, mas que também não supre todas as dificuldades dos universitários, sugerindo o aprimoramento de procedimentos.

\section{REFERÊNCIAS}

Bandeira, M., \& Quaglia, M. A. C. (2005). Habilidades sociais de estudantes universitários: Identificação de situações sociais significativas. Interação em Psicologia, 9(1), 45-55.

Bandeira, M., Costa N. M., Del Prette Z. A. P., Del Prette, A., \& Gerk-Carneiro, E. (2000). Qualidades psicométricas do Inventário de Habilidades sociais (IHS): Estudo sobre a estabilidade temporal e a validade concomitante. Estudos de Psicologia, $5(2), 401-419$.

Boas, A. C. V. V., Silveira, F. F., \& Bolsoni-Silva, A. T. (2005). Descrição de efeitos de um procedimento de intervenção em grupo com universitários: Um estudo piloto. Interação em Psicologia, 9(2), 321-330.

Bolsoni-Silva, A. T. (2009a). Habilidades sociais de universitários: Procedimentos de intervenção na perspectiva da análise do comportamento. Em R. C. Wielenska (Org.), Sobre comportamento e cognição: Vol. 23. Desafios, soluções e questionamentos (pp. 21-52). Santo André: ESETec.

Bolsoni-Silva, A. T. (2009b). Como enfrentar os desafios da universidade. São Carlos: Suprema.

Bolsoni-Silva, A. T., de Paiva, M. M., \& Barbosa, C. G. (2009). Problemas de comportamento de crianças/adolescentes e dificuldades de pais/cuidadores: Um estudo de caracterização. Psicologia Clínica, 21(1), 169-184.

Bolsoni-Silva, A. T., Bitondi, F., \& Marturano, E. M. (2008). Intervenção em grupo para pais: A importância do diagnóstico comportamental individual. Em R. Cavalcanti (Org.), Análise do comportamento: Avaliação e intervenção (pp. 79-100). São Paulo: Roca.

Bolsoni-Silva, A. T., Del Prette, Z. A. P., Del Prette, G., Montanher, A. R. P., Bandeira, M., \& Del Prette, A. (2006). A área das habilidades sociais no Brasil: Uma análise dos estudos publicados em periódicos. Em M. Bandeira, Z. A. P. Del Prette \& A. Del Prette, (Orgs.), Estudos sobre habilidades sociais e relacionamento interpessoal (pp. 17- 45). São Paulo: Casa do Psicólogo.

Botvin, G. J., \& Wills, T. A. (2008). Personal and social skills training: Cognitive-behaviorial approaches to substance abuse prevention. Em C. S. Bell \& R. Battjes (Orgs.), Prevention research: Deterring drug abuse among children and adolescents (pp. 8-49). Washington, DC: U. S. Government Printing Office.

Cole, D. A., Lazarick, D. L., \& Howard, G. S. (1982). Construct validity and the relation between depression and social skill. Journal of Counseling Psychology, 34(3), 315-321.

Cozby, P. C. (2003). Métodos de pesquisa em ciências do comportamento. São Paulo: Atlas.
Del Prette, A., \& Del Prette, Z. A. P. (1983). Análise de repertório assertivo em estudantes de psicologia. Revista de Psicologia, l(1), 15-24.

Del Prette, A., \& Del Prette, Z. A. P. (2001a). Psicologia das relações interpessoais: Vivencias para o trabalho em grupo. Petrópolis: Vozes.

Del Prette, A., \& Del Prette, Z. A. P. (2003). No contexto da travessia para o ambiente de trabalho: Treinamento em habilidades sociais com universitários. Estudos de Psicologia (Natal), 8(3), 413-420.

Del Prette, Z. A. P., \& Del Prette, A. (1999). Psicologia das habilidades sociais: Terapia e educação. Petrópolis: Vozes.

Del Prette, Z. A. P., \& Del Prette, A. (2001b). Inventário de habilidades sociais (IHS-Del-Prette): Manual de aplicação, apuração e interpretação. São Paulo: Casa do Psicólogo.

Del Prette, Z. A. P., Del Prette, A., \& Barreto, M. C. M. (1998). Análise de um inventário de habilidades sociais (IHS) em uma amostra de universitários. Psicologia: Teoria e Pesquisa, 14(3), 219-228.

Del Prette, Z. A. P., Del Prette, A., Barreto, M. C. M., Bandeira, M., Rios-Saldaña, M. R., Ulian, A. L. A. O., Gerk-Carneiro, E., Falcone, E. M. O., \& Villa, M. B. (2004). Habilidades sociais de estudantes de psicologia: Um estudo multicêntrico. Psicologia: Reflexão e Crítica, 17(3), 341-350.

Falcone, E. (1999). Avaliação de um programa de treinamento de empatia com universitários. Revista Brasileira de Terapia Comportamental e Cognitiva, 1(1), 23-32.

Furtado, E. S., Falcone, E. M. O., \& Clark, C. (2003). Avaliação do estresse e das habilidades sociais na experiência acadêmica de estudantes de medicina de uma universidade do Rio de Janeiro. Interação em Psicologia, 7(2), 43-51.

Glasgow, R. E., \& Caul, K. D. M. (2008). Social and personal skills training programs for smoking prevention: Critique and directions for future research. Em C. S. Bell \& R. Battjes (Orgs.), Prevention research: Deterring drug abuse among children and adolescents (pp. 50-66). Washington, DC: U. S. Government Printing Office.

Glass, R. M., \& Kellner, R. (1987). The value of self-report assessment in studies of anxiety disorders. Journal of Clinical Psychopharmacology, 7(4), 215-221.

Gorecki, P., Dickson, A. L., Anderson, H. N., \& Jones, G. E. (1981) Relationship between contrived in vivo and role-play assertive behavior. Journal of Clinical Psychology, 37(1), 104107.

Gorman, D. M. (1996). Do school-based social skills training programs prevent alcohol use among young people? Addiction Research \& Theory, 4(2), 191-210.

Herbert, J. D., Rheingold, A. A., \& Goldstein, S. G. (2002). Brief cognitive behavioral group therapy for social anxiety disorder. Cognitive and Behavioral Practice, 9(1), 1-8.

Inglés, C. J., Hidalgo, M. D., \& Méndez, F. X. (2005). Interpersonal difficulties in adolescence: A new self-report measure. European Journal of Psychologycal Assessment, 21(1), 11-22.

Magalhães, P. P., \& Murta, S. G. (2003). Treinamento de habilidades sociais em estudantes de psicologia: Um estudo préexperimental. Temas em Psicologia, 11(1), 28-37. 
Mersch, P. P. A. (1992). Somatic symptoms in social phobia: A treatment method based on rational emotive therapy and paradoxical interventions. Journal of Behavior Therapy and Experimental Psychiatry, 23(3), 199-211.

Norton, P. J., \& Hope, D. A. (2001). Kernels of truth or distorted perceptions: Self and observer ratings of social anxiety and performance. Behavior Therapy, 32(4), 765-786.

Pacheco, P., \& Rangé, B. P. (2006). Desenvolvimento de habilidades sociais em graduandos de psicologia. Em M. Bandeira, Z. A. P. Del Prette \& A. Del Prette, (Orgs.), Estudos sobre habilidades sociais e relacionamento interpessoal (pp. 199-216). São Paulo: Casa do Psicólogo.

Snaith, R. P. (1981). Rating scales. British Journal of Psychiatry, $138,512-514$.
Welter, G. M., \& Capitão, C. G. (2007) Medidas ipsativas na avaliação psicológica. Avaliação Psicológica, 6(2), 157-165.

Wlazlo, Z., Schroeder-Hartwig, K., Hand, I., Kaiser, G., \& Münchau, N. (2005). Exposure in vivo vs social skills training for social phobia: Long-term outcome and differential effects. Behaviour Research and Therapy, 33(3), 259-269.

Recebido: 10/03/2009 Última revisão: 05/10/2009 Aceite final: 24/11/2009

\section{Sobre os autores:}

Alessandra Turini Bolsoni-Silva: Doutora, docente do Departamento de Psicologia da Universidade Estadual Paulista (UNESP), Bauru/SP e do Programa de Pós-Graduação em Psicologia do Desenvolvimento e Aprendizagem da Universidade Estadual Paulista (UNESP).

Vanessa Barbosa Romera Leme: Doutoranda do Programa de Pós-Graduação em Psicologia da Universidade de São Paulo (FFCLUSP), Ribeirão-Preto/SP.

Anne Midori Abe de Lima: Formada em Psicologia pela Universidade Estadual Paulista (UNESP), Bauru/SP.

Florêncio Mariano da Costa-Júnior: Discente do Programa de Programa de Pós Graduação em Psicologia do Desenvolvimento e Aprendizagem da Universidade Estadual Paulista (UNESP), Bauru/SP.

Marta Regina Gonçalves Correia: Discente do Programa de Programa de Pós Graduação em Psicologia do Desenvolvimento e Aprendizagem da Universidade Estadual Paulista (UNESP), Bauru/SP.

Endereço para correspondência: Alessandra Turini Bolsoni-Silva - Av. Eng. Luiz Edmundo C. Coube, s/n - 17033-360 Bauru/SP Endereço eletrônico: bolsoni@fc.unesp.br. 